\title{
Positron Annihilation Lifetime Measurement of Electron Irradiated Fe-Cr-Ni-P Alloy
}

\author{
By Hideo Watanabe*, Eiichi Kuramoto** and Naoaki Yoshida**
}

\begin{abstract}
$\mathrm{Fe}-16 \% \mathrm{Cr}-17 \% \mathrm{Ni}-0.1 \% \mathrm{P}$ alloy specimens were irradiated with $28 \mathrm{MeV}$ electrons to a dose of $6.0 \times 10^{22} \mathrm{e} / \mathrm{m}^{2}$ at $77 \mathrm{~K}$ and the positron annihilation lifetime measurements were carried out to obtain the isochronal annealing behaviour of radiation-induced defects above room temperature. The three component analysis of lifetime spectra showed that the decrease of second component intensity $I_{2}$ occurred at about $373 \mathrm{~K}$ and $550 \mathrm{~K}$, which are considered to be due to disappearance of mono-vacancies by two different processes, namely, recombination with migrating self-interstitial atoms (SIA's) (373 K) and long range migration of mono-vacancies to sinks $(550 \mathrm{~K})$. This latter stage is about $80 \mathrm{~K}$ higher than the corresponding stage in $\mathrm{Fe}-13 \% \mathrm{Cr}-14 \% \mathrm{Ni}$ alloy specimen, which must be due to the vacancy-phosphorus interaction. The third component (microvoid component) intensity has a peak at about $373 \mathrm{~K}$ and disappeared below $500 \mathrm{~K}$.
\end{abstract}

(Received August 8, 1987)

Keywords: iron-chromium-nickel-phosphorus alloy, electron irradiation, positron annihilation lifetime measurement, vacancy-phosphorus interaction

\section{Introduction}

In the development of structural materials used in the heavy irradiation environments, e.g., fast breeder reactors, fusion reactors and so on, void swelling and radiation embrittlement are the most severe problems which must be attacked. It has recently been recognized that phosphorus-modified stainless steels show a remarkable suppression of void swelling and have become one of the most promising candidate materials ${ }^{(1)(2)}$. On the other hand, it is known that phosphorus atoms segregate to grain boundaries during irradiation and have detrimental effects on mechanical and other physical properties, e.g., fracture toughness. hydrogen embrittlement and stress corrosion cracking $^{(3)}$. In order to understand the effect of phosphorus doping on these properties under heavy irradiation it is definitely necessary to have detailed information on the interaction between phosphorus atoms and radiation-in-

* Graduate Student, Department of High Energy Engineering Science, Interdisciplinary Graduate School of Engineering Sciences, Kyushu University, Kasuga, Fukuoka 816, Japan.

* Research Institute for Applied Mechanics, Kyushu University, Kasuga, Fukuoka 816, Japan. duced point defects.

Positron annihilation technique has become a powerful tool for the investigation of various defects in crystals, especially vacancy type defects, interstitial clusters and dislocations. In the present study attempts have been made to clarify the fundamental aspects of the interaction between phosphorus atoms and radiationinduced defects in $\mathrm{Fe}-\mathrm{Cr}-\mathrm{Ni}-\mathrm{P}$ alloy specimens by means of the positron annihilation lifetime measurement.

\section{Experimental Procedures}

High-purity model austenitic stainless steel $\mathrm{Fe}-16 \% \mathrm{Cr}-17 \% \mathrm{Ni}-0.1 \% \mathrm{P}$ specimens were prepared by high frequency induction melting in flowing purified hydrogen gas atmosphere. The obtained rod was cold rolled into the sheet of thickness of $0.2 \mathrm{~mm}$. The specimens were solution-treated for $1.8 \mathrm{ks}$ at $1323 \mathrm{~K}$ in a vacuum of about $1 \times 10^{-4} \mathrm{~Pa}$.

The electron irradiation of specimens was carried out at $77 \mathrm{~K}$ by using KURRI (Kyoto University Research Reactor Institute) LINAC $(28 \mathrm{MeV})$ to a dose of $6 \times 10^{22} \mathrm{~m}^{-2}$. The concentration of radiation-induced defects was estimated as about 100 appm by the increase of electrical resistivity of high-purity iron mon- 
itor specimen irradiated at the same time. The isochronal annealings were performed for 1.2 $\mathrm{ks}$ from room temperature to $873 \mathrm{~K}$ in steps of $50 \mathrm{~K}$.

Positron annihilation lifetime spectra were obtained with a conventional fast-slow timing spectrometer using ORTEC 473A constant fraction discriminators and RCA 31024A phototubes. The time resolution (FWHM) was $235 \mathrm{psec}$ for ${ }^{60} \mathrm{Co}$. The lifetime spectra were decomposed into three components, namely, a short-lived one $\left(\tau_{1}, I_{1}\right)$ from positrons in bulk material, a longer one $\left(\tau_{2}, I_{2}\right)$ from trapping at vacancies or dislocations (loops) and the longest one $\left(\tau_{3}, I_{3}\right)$ from positrons trapped in microvoids, where $\tau_{i}$ and $I_{i}$ are lifetime and its intensity, respectively. It is rather unfortunate that positron lifetime at dislocations or dislocation loops is slightly shorter than or equal to that at vacancies ${ }^{(4)}$. Dislocations constituting stacking fault tetrahedra must be also taken into account in the case of fcc metals and alloys with low stacking fault energy such as stainless steels. The source components (very long one) which mainly come from thin Mylar foils sandwiching the ${ }^{22} \mathrm{Na}$ positron source were subtracted before three component decomposition. The lifetime spectrum $S(t)$ can be expressed by

$$
\begin{aligned}
S(t)= & \left(I_{1} / \tau_{1}\right) \exp \left(-t / \tau_{1}\right)+\left(I_{2} / \tau_{2}\right) \exp \left(-t / \tau_{2}\right) \\
& +\left(I_{3} / \tau_{3}\right) \exp \left(-t / \tau_{3}\right) .
\end{aligned}
$$

The experimentally observed spectrum is given by a convolution with a time resolution function (Gaussian type) of the apparatus. A least square fitting with experimentally obtained lifetime spectrum was carried out in a large capacity computer to get the best values of each parameter. Details of three components analysis were described in ref. 5 .

\section{Results and Discussion}

Figure 1 shows a positron annihilation lifetime spectrum obtained at room temperature of the $\mathrm{Fe}-16 \% \mathrm{Cr}-17 \% \mathrm{Ni}-0.1 \% \mathrm{P}$ alloy specimen irradiated at $77 \mathrm{~K}$ with $28 \mathrm{MeV}$ electrons to a dose of $6 \times 10^{22} \mathrm{~m}^{-2}$, compared with that of the unirradiated one. It is clearly seen

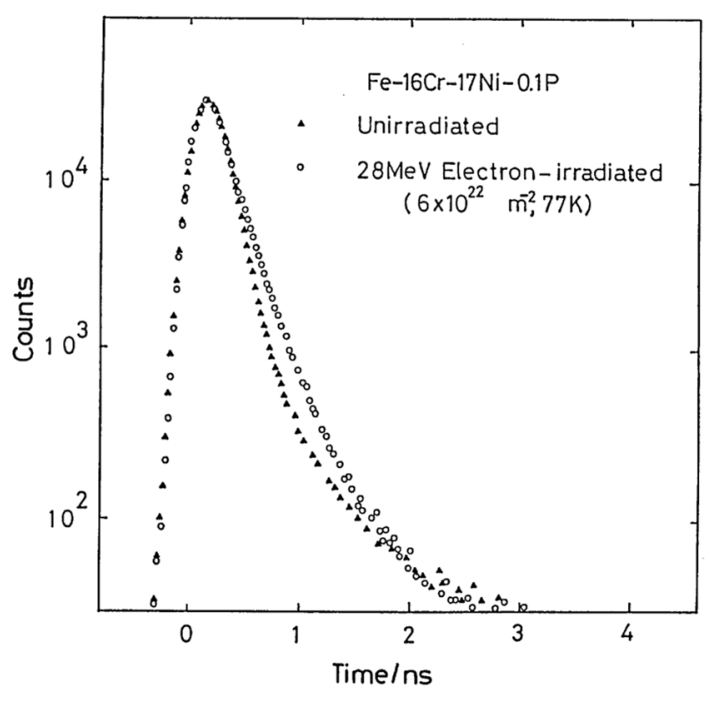

Fig. 1 Positron annihilation lifetime spectra of unirradiated and $28 \mathrm{MeV}$ electron-irradiated $\mathrm{Fe}-16 \% \mathrm{Cr}-$ $17 \% \mathrm{Ni}-0.1 \% \mathrm{P}$ alloy specimen.
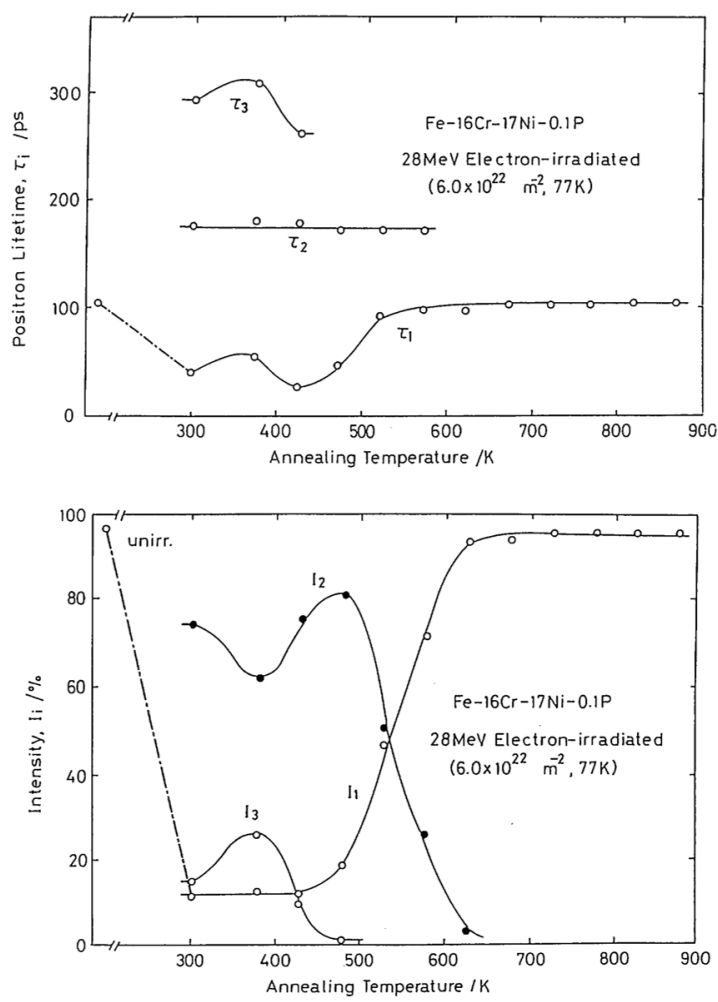

Fig. 2 Isochronal annealing behavior of positron lifetime parameters in $28 \mathrm{MeV}$ electron-irradiated $\mathrm{Fe}-16 \% \mathrm{Cr}-$ $17 \% \mathrm{Ni}-0.1 \% \mathrm{P}$ alloy specimen. 
that the irradiation made the spectrum broader, which indicates that the low temperature electron irradiation introduced defects, especially, vacancies into the specimen. In Fig. 2 are shown the results of three component analysis after each isochronal annealing, namely, the change in lifetimes and their intensities with increase of annealing temperature.

For comparison, Fig. 3 shows the result obtained in the $\mathrm{Fe}-13 \% \mathrm{Cr}-14 \% \mathrm{Ni}$ alloy specimen irradiated under the same condition as above, details of which were presented elsewhere by Kuramoto et al. ${ }^{(6)}$. The grain size of this specimen is about $2 \times 10^{-5} \mathrm{~m}$ which is small compared with the $\mathrm{Fe}-\mathrm{Cr}-\mathrm{Ni}-\mathrm{P}$ alloy $\left(5 \times 10^{-5} \mathrm{~m}\right)$. The reason for the residual value of $I_{2}$ in Fig. 3 at higher temperatures $(>700 \mathrm{~K})$ is a higher trapping probability of positrons at grain boundaries because of the small grain size of this specimen. The accuracy of results from three component analysis is about the size of each circle in the figure except for the
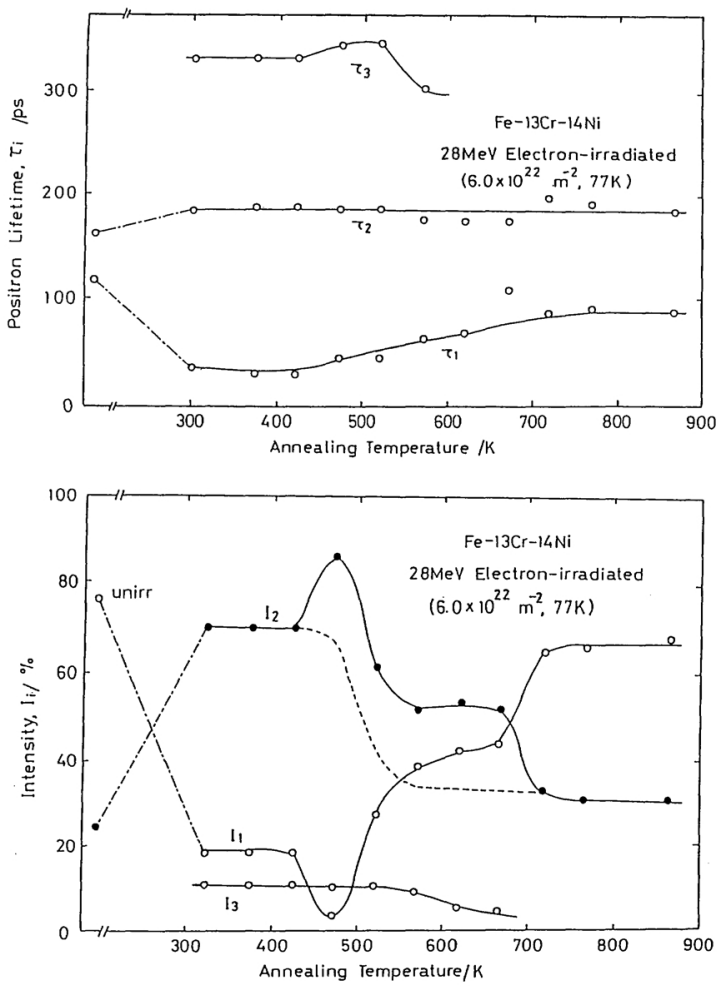

Fig. 3 Isochronal annealing behavior of positron lifetime parameters in $28 \mathrm{MeV}$ electron-irradiated $\mathrm{Fe}-13 \% \mathrm{Cr}-$ $14 \% \mathrm{Ni}$ alloy specimen. case of very low intensity. For the low intensity component $I_{\mathrm{i}}$, the value of lifetime $\tau_{\mathrm{i}}$ was fixed to the value just before the intensity vanished because of significant error of $\tau_{\mathrm{i}}$ above $673 \mathrm{~K}$.

As seen in Fig. 2, the increase and decrease of second component $I_{2}$ were observed. The increase of the second component at about $423 \mathrm{~K}$ is probably due to the growth of the small interstitial clusters to those of the observable size by positron annihilation technique, because SFT's are not observed in the electron irradiation experiment in HVEM (high voltage electron microscope). The decrease of $I_{2}$ at about $373 \mathrm{~K}$ and $550 \mathrm{~K}$ is explained as follows. Dimitrov et al. pointed out that self-interstitial atoms (SIA's) are more mobile than vacancies in $\mathrm{Fe}-\mathrm{Cr}-\mathrm{Ni}$ alloys $^{(7)}$. Moreover, in the previous papers ${ }^{(8)(9)}$ on the HVEM work of this alloy, it was shown that interstitial atoms can migrate around room temperature. Therefore, it is reasonable to consider that these stages are due to disappearance of mono-vacancies by two different processes. Namely, the first one $(373 \mathrm{~K})$ must be due to the recombination with long range migrating SIA's and the second one $(550 \mathrm{~K})$ must be then due to long range migration of mono-vacancies themselves. In the second stage not only vacancies but also SFT's and small interstitial clusters might be included. The corresponding long range vacancy migration stage in $\mathrm{Fe}-13 \% \mathrm{Cr}-14 \% \mathrm{Ni}$ alloy specimen can be found about $473 \mathrm{~K}$ as shown from the dotted line in Fig. 3. In the figure, the solid line (experimentally obtained) is considered to be the summation of the monovacancy component and other components which gives the experimentally obtained increase in $I_{2}$ in the temperature range between $473 \mathrm{~K}$ and $700 \mathrm{~K}$, and then the dotted line in the second component $I_{2}$, the result of subtraction of this increase from the value of solid line in each temperature, shows the simple recovery behaviour of mono-vacancies. There are some reasons which can explain this increase of $I_{2}$, e.g., (i) some of mono-vacancies, which are already mobile, agglomerate to form stacking fault tetrahedra (SFT) and this conversion from mono-vacancies to SFT might give higher trapping rate of positrons because of comparatively large length of resultant dislocation 
lines constituting SFT's, and (ii) growth of interstitial clusters beyond a certain critical size which are large enough to be detected by positron technique. Probably both of these might explain the actual recovery curve of $I_{2}$ experimentally obtained. The comparison between Figs. 2 and 3 indicates that the doping of phosphorus causes the trapping of migrating vacancies, resulting in shift of migration stage to higher temperature by about $80 \mathrm{~K}$.

The third component $I_{3}$ in Fig. 2 showed a peak at about $373 \mathrm{~K}$ and disappeared below $500 \mathrm{~K}$. This temperature range was corresponding to the range where decrease of $\tau_{3}$ was seen. This decrease of $\tau_{3}$ was also observed in Fig. 3 at the temperature of disappearance of $I_{3}$, about $600 \mathrm{~K}$. This means that size of microvoids decreases just before the disappearance by thermal evaporation. But the details of this phenomenon is not fully understood. It is well recognized that long lifetime comes from positrons which annihilated in three dimensional agglomerates of vacancies (microvoids). In specimens irradiated by $28 \mathrm{MeV}$ electrons some amount of cascades must exist and microvoids might be formed without any thermal migrating of vacancies (athermal formation). It is not so easy to explain the existence of a peak in $I_{3}$ in Fig. 2, but one possibility is that the vacancies in cascades migrate in short range to form additional microvoids. If so, in the decrease of $I_{2}$ (first one at about $373 \mathrm{~K}$ mentioned above) the decrease in vacancy concentration via this process must be included. Moreover, there might be a possibility of additional formation of microvoids due to divacancy migration around this temperature $(373 \mathrm{~K})$.

From our previous studies with use of $\operatorname{HVEM}^{(8)(9)}$, it was found that, by phosphorus doping, the peak temperature for the density of vacancy loop was shifted upward by $100 \mathrm{~K}$, as shown in Fig. 4. This is considered to be due to the interaction between vacancies and phosphorus atoms, which is consistent with the present result by positron annihilation experiment.

The migration energy of point defects in $\mathrm{Fe}-$ $16 \% \mathrm{Cr}-17 \% \mathrm{Ni}-0.1 \% \mathrm{P}$ can be estimated by assuming a jump number $N$ in a relation

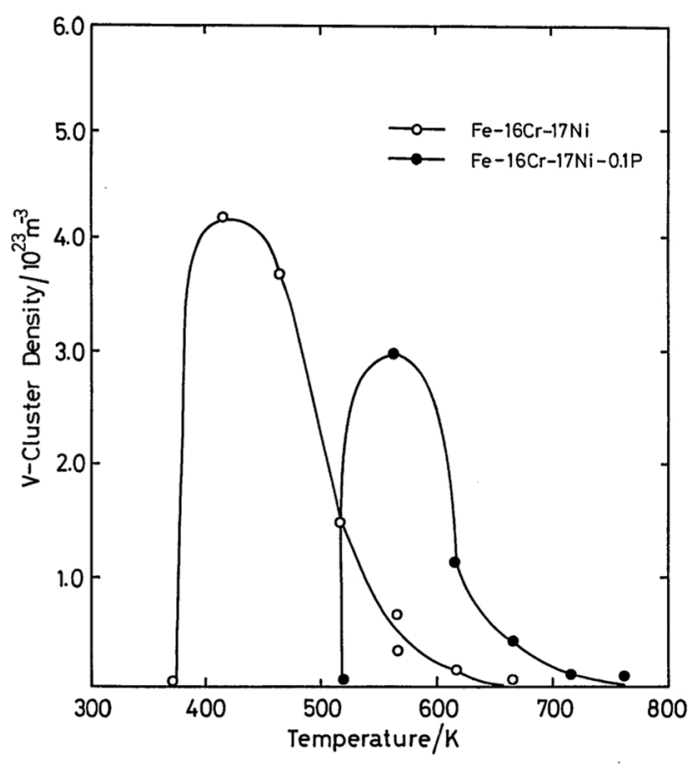

Fig. 4 Temperature dependence of the vacancy type clusters (loops and SFT) density in $\mathrm{Fe}-16 \% \mathrm{Cr}-17 \% \mathrm{Ni}$ and $\mathrm{Fe}-16 \% \mathrm{Cr}-17 \% \mathrm{Ni}-0.1 \% \mathrm{P}$ alloys specimens irradiated to a dose of about $1 \times 10^{26} \mathrm{~m}^{-2}$.

$N=t v_{0} \exp (-E / k T)$, where $v_{0}$ is a jump frequency $\left(\sim 10^{13} \mathrm{~s}^{-1}\right), t$ is the annealing time, $T$ is the annealing temperature, $E$ is migration energy, $k$ is the Boltzmann constant. Considering the sink density in this specimen, we can assume that $N$ is $10^{3}$. With this assumption, migration energy of SIA and vacancy, $E_{\mathrm{m}}^{\mathrm{i}}$ and $E_{\mathrm{m}}^{\mathrm{v}}$, are estimated to be $0.9 \mathrm{eV}^{\dagger}$ and $1.3 \mathrm{eV}$, respectively. The migration energy of SIA $(0.9$ $\mathrm{eV}$ ) is almost consistent with the values which are obtained from HVEM experiments ${ }^{(8)-(10)}$ and the electrical resistivity measurement ${ }^{(7)}$.

\section{Acknowledgment}

This study was made under the Visiting Researcher's Program of Kyoto University Research Reactor Institute (KURRI). The authors express their thanks to Prof. $\mathrm{H}$. Yoshida of KURRI for the electron irradiation experiment and to staffs in HVEM Lab. in Kyushu University for HVEM irradiation experiment.

$\dagger 1 \mathrm{eV}=1.60 \times 10^{-19} \mathrm{~J}(=96.5 \mathrm{~kJ} / \mathrm{mol})$. 


\section{REFERENCES}

(1) E. H. Lee, L. K. Mansur and A. F. Rowcliffe: J. Nucl. Mater., 122/123 (1984), 299.

(2) M. Terasawa, M. Shimada, T. Kakuma, T. Yukitoshi, K. Shiraishi and K. Uematsu: Radiation Effects in Breeder Reactor Structural Materials, Eds. by M. L. Bleiberg and J. W. Bennett, TMSAIME(1977), p. 687.

(3) J. F. Bates, R. W. Rowell and E. R. Gilbert: Effects of Radiation on Materials, Eds. by D. Kramer. H. R. Brager and J. S. Perrin. Tenth Conference, ASTM STP, 725 (1980), 713.

(4) P. Hautojarvi, J. Johansson, T. Judin, P. Moser, M. Puska, A. Vehanen and J. Yli-Kauppila: Proc. 5th Int. Conf. Positron Annihilation, Eds. by R. R.
Hasiguti and K. Fujiwara, (1979), p. 737.

(5) E. Kuramoto and K. Kitajima: Rep. Res. Inst. Appl. Mech. Kyushu Univ., 26 (1978), 11.

(6) E. Kuramoto, N. Kikuchi, D. Irisawa, M. Takenaka and M. Hasegawa: Rep. Res. Inst. Appl. Mech. Kyushu Univ., 65 (1987), 339.

(7) O. Dimitrov and C. Dimitrov: J. Nucl. Mater., 105 (1982), 39.

(8) H. Watanabe, A. Aoki, H. Murakami, T. Muroga and N. Yoshida: J. Japan Inst. Metals, 52 (1988), 536.

(9) H. Watanabe, A. Aoki, H. Murakami, T. Muroga and N. Yoshida: to be published in J. Nucl. Mater.

(10) N. Yoshida, H. Murakami and T. Muroga: International Symposium on In Situ Experiments with HVEM, Ed. by H. Fujita, Osaka Univ., (1985), p. 225. 